\title{
Dimensions of Skill Use in the Unified Protocol: Exploring Unique Effects on Anxiety and Depression
}

\author{
Matthew W. Southward \& Shannon Sauer-Zavala \\ Department of Psychology, University of Kentucky
}

\begin{abstract}
Author Note
Matthew W. Southward: https://orcid.org/0000-0002-5888-2769

Shannon Sauer-Zavala: https://orcid.org/0000-0002-7322-983X
\end{abstract}

Conflicts of interest: The last author receives royalties from Oxford University Press in her role as an author of the Unified Protocol. The first author declares no conflicts of interest.

Acknowledgments: The authors would like to thank Patrick Goh, Caitlyn Hood, Stephen Semcho, and Nicole Stumpp for conducting therapy session and assessments. The authors would also like to thank Destiney MacLean, Anna Garlock, and Alex Urs for their project assistance throughout the study.

Correspondence concerning this article should be addressed to Matthew W. Southward, Department of Psychology, University of Kentucky; 343 Waller Ave., Suite 303, Lexington, KY 40504; Phone: 859-562-1569; Email: southward@uky.edu

\section{In press at Journal of Consulting \& Clinical Psychology}

(C) 2021, American Psychological Association. This paper is not the copy of record and may not exactly replicate the final, authoritative version of the article. Please do not copy or cite without authors' permission. The final article will be available, upon publication, via its DOI: 


\begin{abstract}
Objective: Although the Unified Protocol contains multiple distinct skills to target anxiety, depression, and related conditions, researchers have yet to establish if patients' use of these skills contributes to symptom change. Using data from the first-stage randomization of a sequential multiple assignment randomized trial, we tested whether general skillfulness, defined by skill knowledge, frequency, quality, and effectiveness, predicted within- or between-person changes in anxiety and depression. We further tested whether use of particular UP skills (e.g., mindfulness, behavior change) predicted changes in these outcomes. Method: Participants $(N=$ $70 ; M_{\text {age }}=33.74,67 \%$ female, $74 \%$ white) completed six sessions of core UP modules and reported on their skillfulness and specific skill use using two novel measures, along with their symptoms of anxiety (OASIS) and depression (ODSIS) before each session, and their alliance (WAI) after each session. We disaggregated between- from within-person effects to test sessionto-session effects of skillfulness, skill use, and the alliance on symptom change. Results: Greater within-person skillfulness predicted decreases in anxiety and depression. More frequent withinperson use of all UP skills predicted decreases in anxiety, whereas more frequent within-person use of alternative actions specifically predicted decreases in depression. Conclusions: This pattern of results provides guidance on the specific aspects of skill training to prioritize in transdiagnostic treatments depending on whether therapists are targeting anxiety or depression. Keywords: skills; Unified Protocol; depression; anxiety; alliance
\end{abstract}

Public Health Significance Statement: For people with anxiety and depressive disorders being treated with the Unified Protocol, using therapy skills more skillfully and more frequently than normal predicts decreases in anxiety. Using therapy skills more skillfully and specifically using alternative actions to avoidant urges more frequently than normal predicts decreases in depression. 


\section{Dimensions of Skill Use in the Unified Protocol: Exploring Unique Effects on Anxiety and Depression}

The Unified Protocol for Transdiagnostic Treatment of Emotional Disorders (UP; Barlow et al., 2018) is a modular, cognitive-behavioral treatment (CBT) for anxiety, depressive, and related disorders. The UP is a generally efficacious treatment for these conditions, exerting medium-to-large sized effects (Cassiello-Robbins et al., 2020; Sakiris \& Berle, 2019). The UP's transdiagnostic approach targeting shared maintaining factors across conditions is in line with recent calls for more functional, process-based interventions (Hofmann \& Hayes, 2019). There is preliminary evidence that some transtheoretical processes, such as hope (Gallagher et al., 2020) and experiential avoidance (Eustis et al., 2020), are associated with changes in anxiety in the UP, whereas others, such as the therapeutic alliance (Sauer-Zavala et al., 2018), are not.

The UP consists of five core modules (Wilamowska et al., 2010), each of which teaches patients specific skills to use between sessions. These cognitive, behavioral, and third-wave skills are theorized to contribute to symptom reductions by targeting unique aspects of patients' tendencies to avoid negative emotions. However, the effects of these skills on session-to-session symptom changes in the UP have only been tested in one study of a single patient (Boswell et al., 2014). Thus, it is unclear if the primary activity occurring between sessions (i.e., skill use) is an active mechanism by which the UP influences symptom change. Testing the effect of skill use on symptom changes relative to other influential effects such as the alliance (Flückiger et al., 2018) can clarify which transtheoretical processes actively contribute to symptom change.

\section{Dimensions of Skill Use}

Skill use can be operationalized along several dimensions, including degree of skill knowledge; frequency, quality, and effectiveness of skill use; and the number and type of unique 
skills used (Southward et al., 2021b). Each of these dimensions provides unique information about which aspect of skill use is most important to prioritize in any skill-based treatment, including the UP, to optimize its efficacy and efficiency.

\section{Degree of Skill Knowledge}

To effectively implement skills, patients must first be trained how to use them. The UP has built-in knowledge quizzes included at the end of each module to evaluate how well patients understand the content of the module and the skills therein. In a feasibility study in which patients received UP modules in personalized sequences, patients scored relatively high on these quizzes (average score $=87 \%$; Sauer-Zavala, Cassiello-Robbins, et al., 2019). Patients in this study also demonstrated large-sized decreases in anxiety and depressive symptoms across treatment. Together, these results suggest that UP skills can be effectively taught and can lead to substantial symptom reductions, although it remains unclear if there is a specific relation between skill knowledge and symptom change.

\section{Frequency of Skill Use}

Once patients have been instructed in how to use skills, they are encouraged to use them frequently in response to stressors. In the UP, patients who used mindfulness skills more frequently during treatment were rated by clinicians as having lower post-treatment global clinical severity (Woods et al., 2020) and anxiety (Sauer-Zavala et al., 2021) but not depression (Sauer-Zavala et al., 2021). Among patients who used maladaptive responses (e.g., self-blame, denial, substance use) more frequently at baseline in the UP, ${ }^{1}$ increases in the frequency with which they used adaptive strategies (e.g., acceptance, cognitive reappraisal) over treatment were

\footnotetext{
${ }^{1}$ Patients in this study were randomized to one of four conditions: UP + venlafaxine, UP + placebo, progressive muscle relaxation + venlafaxine, or progressive muscle relaxation + placebo. However, Conklin et al. (2015) reported no significant differences between treatment conditions on the effect described here.
} 
associated with decreases in anxiety and depression symptoms (Conklin et al., 2015).

\section{Quality and Effectiveness of Skill Use}

Skill use can also vary in terms of quality (i.e., how well they are used or how closely their use aligns with how they are taught) and effectiveness (i.e., the degree to which skills lead to intended outcomes, such as short-term reductions in distress; Southward et al., 2021b). Although no researchers to our knowledge have assessed these dimensions of skill use in the UP, preliminary evidence of their effects has been demonstrated in other cognitive and behavioral treatments (see Southward et al. [2021b] for a review). For instance, improvements in the quality of patients' cognitive skills were associated with reductions in depressive symptoms across cognitive therapy for depression (Adler et al., 2015) and predicted subsequent reductions in depressive symptoms in internet-delivered CBT for depression (Forand et al., 2018). Southward et al. (2021a) tested the effectiveness of patients' skill use among patients in Dialectical Behavior Therapy (DBT) skills training. In this study, greater daily skill effectiveness was related to less intense daily anxiety and depression. Because patients also reported feeling more effective on days they used more skills, these results suggest that using skills more effectively may predict decreases in anxiety and depression.

\section{Which Skills for Which Outcomes}

Finally, it remains an open question whether certain skills taught in the UP are more strongly predictive of outcomes than others. As noted above, Boswell et al. (2014) found that more frequent use of mindfulness predicted decreases in anxiety and depressive symptoms whereas more frequent use of cognitive reappraisal only predicted decreases in depressive symptoms for a woman with major depressive disorder (MDD) and generalized anxiety disorder (GAD). However, Sauer-Zavala et al. (2021) found that patients who used mindfulness more 
frequently across treatment tended to report lower post-treatment anxiety but not depression. Of course, mindfulness and cognitive reappraisal are only two skills taught in the UP. In an independent idiographic study of a woman with GAD and MDD, the mindfulness module of the UP led to increases in restlessness, the cognitive flexibility module was unrelated to changes in anxiety or depression, and the exposure module led to decreases in avoidance, procrastination, and concentration difficulties (Altman et al., 2020). Although researchers have tested experimental interventions of other UP skills (e.g., countering emotional behaviors; SauerZavala, Wilner, et al., 2019; Sauer-Zavala et al., 2020), these relatively small studies make it difficult to draw strong conclusions about the unique effects of the full suite of UP skills.

\section{Limitations of the Current Literature}

With the exception of Altman et al. (2020) and Boswell et al.'s (2014) idiographic studies, four limitations of existing studies of the UP limit the strength of the conclusions that can be drawn about the effects of UP skills: (1) the variety of UP skills assessed, (2) a lack of explicit disaggregation of between- from within-person effects of skill use, (3) the relative infrequency with which skills and outcomes were measured, and (4) a lack of concurrently modeled alternative mechanisms. First, although researchers have included measures of mindfulness and cognitive reappraisal frequency in several studies, these only represent two of the five core modules of the UP, limiting our understanding of the remaining skills. Second, by not disaggregating between- from within-person effects, it remains unclear how much of these effects on symptom changes are driven by relatively stable individual differences in skill use (between-person effects) and how much is driven by changes in each patient's skill use over treatment (within-person effects). Third, at the end of each session of the UP, therapists assign patients specific skills to practice for homework before the next session. However, the most 
frequent assessment schedule in UP trials was every four sessions, with several studies relying on pre-post assessment schedules. Infrequent assessments render it difficult to establish temporal relations between skill use and symptom change. Moreover, these assessment schedules do not allow researchers to rule out confounding from unmeasured third variables (e.g., therapeutic alliance, external events) that could explain observed effects. Relatedly, most researchers have tested skill use independently of well-studied alternative transtheoretical mechanisms (e.g., therapeutic alliance). Including such alternative mechanisms allows for a stronger test of the relative efficacy of skill use (Southward et al., 2021b).

\section{Current Study}

To clarify the effects of different dimensions of skill use in the UP and address the limitations of previous analyses, we conducted two sets of secondary analyses of data from a randomized clinical trial (NCT04584879; Sauer-Zavala et al., 2021). In Aim 1, we tested the between- and within-person effects of a novel measure of transtheoretical skillfulness on sessionto-session changes in anxiety and depression in the UP. We hypothesized that (1a) our novel measure of transtheoretical skillfulness would exhibit a one-factor structure at the between- and within-person level and (1b) demonstrate convergent validity with validated measures of skill use and discriminant validity with measures of symptom severity; (1c) the UP would lead to significant changes in anxiety and depression; and (1d) greater between- and within-person skillfulness would predict session-to-session reductions in anxiety and depression above and beyond an alternative transtheoretical mechanism (i.e., the working alliance). We also conducted an exploratory analysis to test which of the dimensions of skillfulness led to the greatest between- and within-person session-to-session reductions in these outcomes.

In Aim 2, a subsample of participants completed a novel measure of UP skill use 
indicating the frequency with which each core UP skill was used to test if UP skills demonstrated relatively unique effects on anxiety and depression. We hypothesized that this measure of UP skill use would (2a) exhibit a one-factor structure at the between- and within-person level; (2b) demonstrate convergent validity with measures of skill use and discriminant validity with measures of symptoms. We further hypothesized that (2c) using skills more frequently would predict between- and within-person session-to-session reductions in anxiety and depression over and above the alliance. Finally, we explored which of the core UP skills led to the greatest session-to-session reductions in anxiety and depression.

\section{Methods and Materials}

\section{Participants}

The sample consisted of 70 participants with at least one anxiety, depressive, or related disorder based on Diagnostic and Statistical Manual of Mental Disorders (5th ed.; DSM-5; American Psychiatric Association, 2013) criteria. Participants were 33.74 years old $(S D=12.64)$ on average, with a majority of participants identifying as female $(n=47 ; 67.1 \%)$, White $(n=52$; $74.3 \%)$, and heterosexual $(n=52 ; 74.3 \%)$, with a median household income between $\$ 50,000$ $\$ 99,999$. Potential participants were eligible for the study if they met criteria for at least one of the following disorders: generalized anxiety disorder (GAD; $n=33 ; 47.1 \%)$, major depressive disorder (MDD; $n=19 ; 27.1 \%)$, social anxiety disorder (SAD; $n=16 ; 22.9 \%)$, persistent depressive disorder (PDD; $n=12 ; 17.1 \%$ ), obsessive-compulsive disorder (OCD; $n=5 ; 7.1 \%)$, panic disorder (PD; $n=4 ; 5.7 \%)$, posttraumatic stress disorder (PTSD; $n=3 ; 4.3 \%)$, acute stress disorder (ASD; $n=1 ; 1.4 \%$ ), and agoraphobia (AG; $n=1 ; 1.4 \%$ ). Potential participants were excluded if they exhibited symptoms requiring more intensive treatment (i.e., mania in the past year; acute suicidality; substance use disorder in the past three months; or psychotic features); 
had received five or more sessions of CBT in the past five years; were unwilling to stop concurrent treatment for an emotional disorder; or were unwilling to maintain a stable dosage of medication during study participation. The study was approved by the local Institutional Review Board, and all participants provided informed consent before engaging in study procedures.

\section{Study Treatment}

The treatment consisted of the five core modules of the UP, delivered individually. Each module, except Countering Emotional Behaviors, was delivered once per week over two 50-60 minute sessions. Countering Emotional Behaviors was delivered in an individual format once per week over four 50-60 minute sessions. The Understanding Emotions module teaches patients to notice the connections among their thoughts, emotions, physical sensations, and behaviors and the short- and long-term effects of these connections. The Mindful Emotion Awareness module teaches patients to nonjudgmentally observe their present-moment experiences. The Cognitive Flexibility module teaches patients to generate alternative interpretations of or explanations for ineffective thoughts. The Countering Emotional Behaviors module teaches patients to practice behaviors that counteract ineffective, mood-congruent behavioral urges. Finally, the Confronting Physical Sensations module teaches patients to practice exposure exercises to tolerate uncomfortable physical sensations. Four therapists (a licensed clinical psychologist, a postdoctoral fellow, and two advanced clinical psychology graduate students; two men, two women) provided the treatment and demonstrated good adherence (Sauer-Zavala et al., 2021).

\section{Measures}

\section{Dimensions of Skillfulness and Use Scale}

Created for the current study, the Dimensions of Skillfulness and Use Scale (DSUS) is a 4-item self-report measure in which each item is designed to assess an aspect of skillfulness: 
understanding of skills learned in the prior session, frequency of skill use, quality of skill use (i.e., how well skills were implemented in line with how they were taught regardless of the outcome), and effectiveness (i.e., the degree to which the skill led to desired outcomes; see Supplemental Materials for the full text of all items). Items are rated from 0 (not at all/never) to 4 (extremely/every day). DSUS items demonstrated acceptable-to-good internal consistency across sessions 2-7 (McDonald's $\omega s:$.76-.86).

\section{Unified Protocol Skills Use Scale}

Created for the current study, the Unified Protocol Skill Use Scale (UPSUS) is a 7-item self-report measure designed to assess the frequency with which people use UP skills in distressing situations. Each item was written to describe core skill(s) taught in each of the five core UP modules (Supplemental Materials). Participants rated each item on a 6-point scale, from 0 (Not at all [0\% of emotional experiences]) to 5 (Nearly all the time [90-100\% of emotional experiences ]), with the option to note that they did not know or had not learned the skill marked as a missing response. Among the subsample ${ }^{2}$ of participants who completed it, UPSUS items demonstrated good-to-excellent internal consistency across sessions 2-7 ( $\omega$ s: .83-.92).

\section{Overall Anxiety Severity and Impairment Scale}

The Overall Anxiety Severity and Impairment Scale (OASIS; Norman et al., 2006) is a 5item self-report measure designed to assess the severity of anxiety symptoms over the past week. Items are rated from 0 to 4 with unique anchors for each item and summed to create a total score.

\footnotetext{
${ }^{2}$ A randomly selected subsample of participants $(n=43)$ completed the UPSUS. To reduce participant burden, participants were randomly assigned to complete either the UPSUS or a personality measure before each session of the study. These participants did not differ from the remaining participants on age $(M=33.44, S D=11.42)$, gender $\left(n_{\text {female }}=31 ; 72.1 \%\right)$, racial/ethnic background $\left(n_{\text {White }}=31 ; 72.1 \%\right)$, sexual orientation $\left(n_{\text {heterosexual }}=34 ; 79.1 \%\right)$, median household income $(\$ 75,000-\$ 99,999)$, primary diagnoses $\left(n_{\mathrm{GAD}}=19[44.2 \%] ; n_{\mathrm{MDD}}=11[25.6 \%] ; n_{\mathrm{SAD}}=9\right.$ $\left.[20.9 \%] ; n_{\mathrm{PDD}}=9[20.9 \%] ; n_{\mathrm{OCD}}=3[7.0 \%] ; n_{\mathrm{PD}}=2[4.7 \%] ; n_{\mathrm{PTSD}}=1[2.3 \%] ; n_{\mathrm{AG}}=1[2.3 \%]\right)$, or sequencing condition allocation $\left(n_{\text {capitalization }}=15[34.9 \%] ; n_{\text {compensation }}=16[37.2 \%] ; n_{\text {standard }}=12[27.9 \%]\right), p s>.14$.
} 
OASIS items demonstrated acceptable-to-good internal consistency across the first seven sessions ( $\omega \mathrm{s}: .79-.88)$.

\section{Overall Depression Severity and Impairment Scale}

The Overall Depression Severity and Impairment Scale (ODSIS; Bentley et al., 2014) is a 5-item self-report measure designed to assess the severity of depressive symptoms over the past week. Items are rated from 0 to 4 with unique anchors for each item and summed to create a total score. ODSIS items demonstrated excellent internal consistency across the first seven sessions

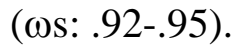

\section{Southampton Mindfulness Questionnaire}

The Southampton Mindfulness Questionnaire (SMQ; Chadwick et al., 2008) is a 16-item self-report measure designed to assess the degree to which a person is mindfully aware of distressing thoughts and images. Items are rated from 0 (disagree totally) to 6 (agree totally) and summed to create a total score. SMQ items demonstrated good-to-excellent internal consistency across the first seven sessions ( $\omega \mathrm{s}: .86-.92)$.

\section{Unified Protocol Cognitive Skills Questionnaire}

The Unified Protocol Cognitive Skills Questionnaire (UP-CSQ; Sauer-Zavala et al., 2017) is a 7-item self-report measure designed to assess how frequently people examined alternative explanations for their initial negative thoughts in the past week. Items are rated from 1 (never) to 5 (always or when needed) and summed to create a total score. UP-CSQ items demonstrated good-to-excellent internal consistency across the first seven sessions ( $\omega \mathrm{s}:$.87-.94).

\section{Working Alliance Inventory - Short Revised}

The Working Alliance Inventory - Short Revised (WAI-SR; Hatcher \& Gillaspy, 2006) is a 12-item self-report measure designed to assess patients' perceptions of the therapeutic 
alliance. Items are rated from 1 (seldom) to 5 (always) and summed to create a total score. WAISR items demonstrated excellent internal consistency across the first six sessions ( $1 \mathrm{~s}:$.91-.95).

\section{Study Design}

The present study is a secondary data analysis of Sauer-Zavala et al. (2021). The parent study was a sequential multiple assignment randomized trial in which participants were first randomized to receive UP modules in one of three sequences: (1) prioritizing modules that capitalized on patients' relative strengths $(n=24 ; 34.3 \%),(2)$ prioritizing modules that compensated for patients' relative deficits $(n=21 ; 30.0 \%)$, or $(3)$ the standard published order of UP modules ( $n=25 ; 35.7 \%$; Barlow et al., 2018). Just before mid-treatment (i.e., between sessions 5 and 6), participants were entered into a second randomization in which half of participants were assigned to discontinue treatment after session 6 (Brief treatment condition) and half were assigned to complete the remaining six sessions (Full treatment condition). Those in the Brief treatment condition continued to fill out all measures above, except the WAI-SR, weekly for six weeks following their final session (session 6).

Participants reported their levels of skillfulness, UP skill use, anxiety, depression, mindfulness, and cognitive flexibility no more than 24 hours prior to the start of each session via a REDCap link. Because participants had not learned any skills prior to their first session, they did not complete the DSUS or UPSUS at this time point. After each session, participants rated the working alliance via a separate REDCap link.

\section{Data Analytic Plan}

\section{Aim 1}

To test our first hypothesis (1a), we conducted a multilevel confirmatory factor analysis (CFA) in Mplus Version 7.0 (Muthén \& Muthén, 1998-2012) using maximum likelihood 
estimation with robust standard errors to explore the between- and within-person factor structure of DSUS items. We evaluated the fit of this model using the chi-squared statistic, root-meansquared error of approximation (RMSEA), comparative fit index (CFI), the Tucker-Lewis index (TLI), and the standardized root-mean-squared residual (SRMR) estimated at each level of the model. Following $\mathrm{Hu}$ and Bentler (1999), the following fit indices indicated excellent model fit: a non-significant chi-squared statistic, RMSEA $<.06$, CFI and TLI $>.95$, and SRMR $<.08$. Models with RMSEA $<.10$ and CFI and TLI > .90 were interpreted to have acceptable model fit.

To evaluate the convergent and discriminant validity of the DSUS (Hypothesis 1b), we tested whether DSUS scores were more strongly correlated with validated measures of skill use (i.e., SMQ, UP-CSQ) than with measures of symptom severity (i.e., OASIS, ODSIS). We used Steiger's (1980) equations to compare two dependent correlations with one variable in common and used the absolute value of all correlations to account for different directions of associations.

To test our third hypothesis (1c), we examined changes over time in anxiety and depression, as well as skillfulness and the alliance. We tested whether these variables changed over the first six sessions using hierarchical linear modeling (HLM) with proc mixed in SAS Version 9.4. We regressed each variable on session number in separate models, entering therapist and sequencing condition as covariates, and including random intercepts and slopes. We focused on the first six sessions for our primary analyses because (a) only half of participants continued to receive treatment after this point and (b) the majority of change in depression and anxiety has been shown to occur over the first six sessions (Niileksela et al., 2021). However, we also conducted supplementary analyses using all available sessions for comparison (Tables S21-S37, Supplemental Materials).

Finally, we tested whether general skillfulness predicted session-to-session changes in 
anxiety and depression over and above the alliance. We first disaggregated participants' DSUS scores into between- and within-person variability in line with Curran and Bauer's (2011) recommendations. ${ }^{3}$ Between-person DSUS scores were determined by (a) calculating each participant's mean DSUS score across sessions 2-7 and (b) calculating a grand mean of the sample from all participants, and (c) subtracting the grand mean from each participant's mean. Within-person DSUS scores were determined by subtracting each participant's mean DSUS score across sessions 2-7 from their raw DSUS score at a given session. We repeated this process for WAI-SR scores at session 1-6. We then regressed the target symptom (e.g., anxiety) at session $t$ on (a) between- and within-person skill use measured at session $t$, (b) between- and within-person alliance at session $t-1$, (c) the target symptom at session $t-1$, (d) the non-target symptoms (e.g., depression) at session $t$, (e) session number, (f) a dummy-coded sequencing condition indicator variable, and (g) a dummy-coded therapist indicator variable using proc mixed. ${ }^{4}$ The fixed effects of this model are specified with the following equation:

$$
\begin{aligned}
\text { OASIS }_{i t}=\gamma_{00} & +\gamma_{01}\left(\text { DSUS }_{i}\right)+\gamma_{10}\left(\text { DSUS }_{i t}\right)+\gamma_{02}\left(\text { WAI }_{i}\right)+\gamma_{20}\left(\text { WAI }_{i t-1}\right)+\gamma_{30}\left(\text { OASIS }_{i t-1}\right) \\
& +\gamma_{40}\left(\text { ODSIS }_{i t}\right)+\gamma_{50}\left(\text { session }_{i t}\right)+\gamma_{03}\left(\text { seq }_{\text {condition }}\right)+\gamma_{04}\left(\text { therapist }_{i}\right) \\
& +e_{i t}
\end{aligned}
$$

We then replaced anxiety with depression in a separate model. We applied restricted maximum likelihood estimation with random intercepts, an autoregressive lag-1 residual covariance structure, and used the Kenward-Roger method to calculate degrees of freedom. We

\footnotetext{
${ }^{3}$ For comparison, models without disaggregation are presented in supplemental materials (Tables S38-S45).

${ }^{4} \mathrm{We}$ included the non-target symptom as a covariate to better specify the effects of skillfulness on the target symptom. We included session number to account for time in line with Wang and Maxwell's (2015) recommendations. We included the dummy-coded sequencing condition indicator variable, using the standard sequencing condition as the reference category, to account for effects of module sequences. We included a dummycoded therapist indicator variable to account for therapist effects rather than a random effect of therapists because, with only four therapists, fixed effects models may be less biased than random effects models (McNeish \& Stapleton, 2016).
} 
used the Glimmix_R2 macro (Jaeger et al., 2017) in SAS to estimate $R^{2}$ effect sizes. In these models, we had $80 \%$ power to detect medium-sized between-person effects $\left(R^{2} \geq .10\right.$; Faul et al., 2009) and small-sized within-person effects $\left(R^{2} \geq .01\right.$; Lafit et al., 2021).

\section{Aim 2 - UP Skill Use}

To test our first hypothesis (2a), we conducted a multilevel CFA in Mplus Version 7.0 (Muthén \& Muthén, 1998-2012) using maximum likelihood estimation with robust standard errors to explore the between- and within-person factor structure of UPSUS items. We evaluated the fit of this model using Hu and Bentler's (1999) criteria above.

To evaluate the convergent and discriminant validity of the UPSUS (Hypothesis $2 b$ ), we tested whether UPSUS scores were more strongly correlated with measures of skill use (i.e., SMQ, UP-CSQ) than with measures of symptom severity (i.e., OASIS, ODSIS). We used Steiger's (1980) equations to compare the absolute value of correlations as in Aim 1.

We calculated and assessed descriptive statistics of the average frequency of UP skill use as well as the frequency with which each specific skill was used. We examined whether the frequency with which UP skills in general, and each UP skill specifically, was used changed over sessions 2-7 with proc mixed using the HLM specifications from Aim 1.

For our primary hypotheses, we tested whether general and specific UP skill frequency predicted session-to-session changes in anxiety and depression. We first disaggregated average UP skill frequency and the frequency of each UP skill into between- and within-person variability using the procedures described in Aim 1. We then conducted the same linear mixed models as in Aim 1, replacing between- and within-person DSUS scores with between- and within-person UPSUS scores. The fixed effects of this model are specified in the equation: 


$$
\begin{aligned}
\text { OASIS }_{i t}=\gamma_{00} & +\gamma_{01}\left(\text { UPSUS }_{i}\right)+\gamma_{10}\left(\text { UPSUS }_{i t}\right)+\gamma_{02}\left(\text { WAI }_{i}\right)+\gamma_{20}\left(\text { WAI }_{i t-1}\right) \\
& +\gamma_{30}\left(\text { OASIS }_{i t-1}\right)+\gamma_{40}\left(\text { ODSIS }_{i t}\right)+\gamma_{50}\left(\text { session }_{i t}\right)+\gamma_{03}\left(\text { seq condition }_{i}\right) \\
& +\gamma_{04}\left(\text { therapist }_{i}\right)+e_{i t}
\end{aligned}
$$

We repeated this process, replacing anxiety and depression, and conducted two further models, replacing UPSUS total scores with all specific UP skills. In these models, we had $80 \%$ power to detect medium-to-large sized between-person effects $\left(R^{2} \geq .16\right.$; Faul et al., 2009) and medium-to-large-sized within-person effects $\left(R^{2} \geq .11\right.$; Lafit et al., 2021). All code is available at: https://osf.io/7k3ay/?view_only=a86dcc04e64d4d67b83f147b70784e57

\section{Results}

\section{Aim 1 - Skillfulness}

A 1-between/1-within factor structure provided excellent fit to the DSUS items across all indices, and all items demonstrated significant, positive factor loadings $>.50$ at both levels (Table 1a). We thus used a sum score of these items for our primary analyses.

We next examined the intraclass correlations (ICCs) of our measures of interest to determine the proportion of variability in each measure attributable to relatively stable betweenperson differences and the proportion attributable to within-person fluctuations. The ICC for the DSUS was .47 , indicating $47 \%$ of the variability in this measure was due to between-person differences and $53 \%$ of the variability was due to within-person fluctuations. Similarly, the ICC for the OASIS was .42, indicating the majority of the variability was within-persons. By contrast, the ICCs for the ODSIS (.60) and WAI-SR (.66) indicated the majority of variability in these measures was between-persons.

There were no significant associations between demographic characteristics and skillfulness, $r \mathrm{~s}<|.12|, p s>.05$ (Table $\mathrm{S} 2$ ), so we did not include demographic characteristics in 
subsequent analyses. Skillfulness exhibited significant positive correlations with between- and within-person cognitive skills and within-person mindfulness, as well as a non-significant positive correlation with between-person mindfulness, demonstrating convergent validity (Table 2a). Skillfulness exhibited significant negative correlations with within-person anxiety and depression, but a non-significant negative correlation with between-person anxiety and a nonsignificant positive correlation with between-person depression (Table 2a). The absolute value of the correlations between skillfulness and cognitive skills, but not mindfulness, were generally greater than those between skillfulness and anxiety $\left(z_{\text {between }}=3.78, p<.01 ; z_{\text {within }}=3.05, p<.01\right)$ and depression $\left(z_{\text {between }}=3.65, p<.01 ; z_{\text {within }}=1.88, p=.06\right)$, demonstrating evidence of discriminant validity. Of note, cognitive skills were more distinct from anxiety and depression than mindfulness (Table 2a).

Despite a numerical increase from sessions 3-6 (Table 3a), skillfulness did not significantly increase from session 2-7, $B=.10, S E=.07, p=.15,95 \% \mathrm{CI}[-.04, .24], R^{2}=.04$. Specifically, although skill quality, $B=.02, S E=.01, p=.047,95 \%$ CI $[<.01, .05], R^{2}=.06$, and effectiveness, $B=.03, S E=.01, p=.02,95 \%$ CI $[.004, .05], R^{2}=.10$, increased, skill knowledge, $B=-.004, S E=.01, p=.72,95 \% \mathrm{CI}[-.03, .02], R^{2}<.01$, and frequency, $B=.02$, $S E=.02, p=.31,95 \% \mathrm{CI}[-.02, .05], R^{2}=.02$, did not. By contrast, anxiety, $B=-.30, S E=.07$, $p<.01,95 \%$ CI $[-.43,-.17], R^{2}=.16$, and depression, $B=-.42, S E=.08, p<.01,95 \%$ CI $[-.59$, -.26], $R^{2}=.11$, significantly decreased, and the alliance significantly increased, $B=1.26, S E=$ $.17, p<.01,95 \%$ CI $[.92,1.59], R^{2}=.66$, over these early sessions (Table 3a).

When examining lagged effects, within-person increases in skillfulness predicted sessionto-session decreases in anxiety, $B=-.15, S E=.08, p=.047,95 \% \mathrm{CI}[-.30,-.001], R^{2}=.02$, and depression, $B=-.20, S E=.07, p=.01,95 \%$ CI $[-.35,-.05], R^{2}=.03$ (Tables S3-S4). Between- 
person skillfulness was not significantly related to each outcome, $p \mathrm{~s}>.15, R^{2}<.07$. Only

between-person ratings of the alliance were significantly associated with decreases in depression, $B=-.11, S E=.05, p=.0497,95 \%$ CI $[-.22,<-.01], R^{2}=.11 ;$ all other effects of the alliance were non-significant, $p \mathrm{~s}>.10, R^{2}<.08$.

When all four dimensions of skillfulness were entered as simultaneous predictors, only within-person increases in knowledge predicted reductions in anxiety, $B=-.66, S E=.29, p=$ $.02,95 \%$ CI $[-1.23,-.09], R^{2}=.02$ (Table S5). No within-person dimensions predicted changes in depression, $p \mathrm{~s}>.20, R^{2}<.01$, and no between-person dimensions were significantly related to changes in anxiety or depression, $p \mathrm{~s}>.10, R^{2}<.08$ (Tables S5-S6). ${ }^{5}$

\section{Aim 2 - Unified Protocol Skill Use}

A 1-between/1-within factor structure provided acceptable-to-excellent fit to the UPSUS items across all indices, and all items demonstrated significant, positive loadings on factors at both levels (Table 1b). We thus used a total score to represent average UP skill frequency in our analyses. The ICCs for UPSUS frequency (.50) and specific skill scores (range: .20-.46) were $\leq$ .50 , indicating that at least half the variability in these measures was within-persons (Table S8).

UPSUS frequency exhibited significant positive correlations with between- and withinperson cognitive skills and within-person mindfulness, as well as a non-significant positive correlation with between-person mindfulness, demonstrating convergent validity (Table 2b). UPSUS frequency exhibited significant negative correlations with within-person anxiety and depression, but non-significant zero or positive correlations with between-person anxiety and depression (Table 2b). The absolute value of the correlations between UPSUS frequency and

\footnotetext{
${ }^{5}$ To examine reverse causality (i.e., symptom change predicting change in skillfulness), we tested between- and within-person anxiety and depression as simultaneous predictors of next-session skillfulness controlling for currentsession skillfulness, session number, sequencing condition, and therapist. No symptom score significantly predicted changes in skillfulness, $p \mathrm{~s}>.05$ (Table S7).
} 
cognitive skills, but not mindfulness, were generally greater than those between UPSUS frequency and depression $\left(z\right.$ between $\left.=2.86, p<.01 ; z_{\text {within }}=1.99, p=.047\right)$ and anxiety $(z$ between $=$ $3.80, p<.01 ; z$ within $=1.82, p=.06)$, demonstrating evidence of discriminant validity.

The average frequency with which participants used UP skills increased across treatment, $B=.10, S E=.03, p<.01,95 \% \mathrm{CI}[.03, .16], R^{2}=.33$, from sometimes $(11-35 \%$ of emotional experiences) to about half the time (36-65\% of emotional experiences). Participants reported significant increases in the frequency with which they used all specific UP skills, $B \mathrm{~s}>.05, p \mathrm{~s}<$ $.03, R^{2}>.20$ (Table $\left.3 b\right)$.

When examining lagged effects, greater within-person UP skill frequency (i.e., using UP skills more frequently than one's personal average) predicted decreases in session-to-session anxiety, $B=-.64, S E=.30, p=.04,95 \%$ CI $[-1.24,-.04], R^{2}=.03$, but not depression, $p=.34$, $R^{2}=.01$ (Tables S9-S10). Between-person UP skill frequency was not significantly related to changes in anxiety and depression, $p \mathrm{~s}>.05, R^{2}<.12$.

When examining individual UP skills, greater within-person use of Countering Emotional Behaviors 2 (practicing adaptive alternative behaviors) significantly predicted session-to-session decreases in depression, $B=-.75, S E=.32, p=.02,95 \% \mathrm{CI}[-1.38,-.12], R^{2}=.11$, whereas greater within-person use of Understanding Emotions 1 (identifying cognitive, physical, and behavioral aspects of emotions) significantly predicted session-to-session increases in depression, $B=.83, S E=.40, p=.04,95 \%$ CI $[.02,1.63], R^{2}=.06$ (Table S12). Within-person changes in these skills did not significantly predict changes in anxiety, $p \mathrm{~s}>.15, R^{2}<.04$, and within-person changes in all other skills did not significantly predict changes in anxiety or depression, $p \mathrm{~s}>.05, R^{2}<.05$. (Tables S11-S12). Between-persons, only greater use of Confronting Physical Sensations (allowing experiences of uncomfortable physical sensations) 
was related to lower average anxiety, $B=-1.35, S E=.59, p=.03,95 \%$ CI $[-2.54,-.15], R^{2}=$ .19 (Table S11). $^{6}$

\section{Discussion}

Using two novel measures of skillfulness and skill use, we found evidence that withinperson changes in skillfulness and use predicted session-to-session changes in anxiety and depression in the UP. Greater transtheoretical skillfulness (i.e., knowledge, frequency, quality, and effectiveness) than one's personal average predicted decreases in anxiety and depression over and above the working alliance. Using a range of UP skills more frequently than one's personal average predicted decreases in anxiety, and more frequently acting counter to one's avoidant urges (i.e., Countering Emotional Behaviors 2) specifically predicted decreases in depression, whereas more frequently identifying thoughts, physical sensations, and behaviors associated with emotions (i.e., Understanding Emotions 1) predicted increases in depression, over and above the alliance.

The results of Aim 1 replicate and extend previous findings regarding symptom change in the UP and extend previous work on the roles of skillfulness and the alliance. The UP led to significant decreases in anxiety and depression symptoms, in line with previous work (Sakiris \& Berle, 2019). Similarly, in line with results from Sauer-Zavala et al. (2018), the UP led to significant increases in the strength of the therapeutic alliance early in treatment. Although the UP led to significant increases in the frequency with which UP skills were used, it did not lead to significant increases in general skillfulness. At the item level, skill quality and effectiveness

\footnotetext{
${ }^{6}$ When testing reverse causality (Tables S13-S20), only two effects of symptoms on changes in skill use were significant. Within-person increases in anxiety predicted session-to-session increases in the use of Countering Emotional Behaviors $1, B=.10, S E=.04, p=.03,95 \%$ CI $[.01, .19], R^{2}=.06$ (Table S18) and within-person increases in depression predicted session-to-session increases in the use of Confronting Physical Sensations, $B=.08$, $S E=.04, p=.03,95 \%$ CI $[.01, .15], R^{2}=.05$ (Table S20).
} 
significantly increased but skill knowledge and frequency did not. Of note, frequency in the DSUS was measured in terms of days whereas frequency in the UPSUS was measured in terms of distressing emotional experiences. These results thus suggest that the UP leads to increases in how well, how effectively, and how frequently patients use skills when needed but does not necessarily lead to improvements in how well patients understand skills or how frequently they use skills in general. These results may be expected, in that as patients experience symptom reduction, they need to use skills less frequently in general, but may use learn to use them more frequently in response to emotionally distressing experiences. The lack of change in understanding the skills may reflect the fact that different skills were taught every 2-4 sessions so patients may not have deepened their understanding of specific skills but maintained a higherthan-average understanding of each skill as it was taught.

Although the UP led to significant improvements in the alliance but not skillfulness across the first 6 sessions, greater within-person skillfulness between each session, and not the alliance, predicted small-sized reductions in anxiety and depression. These results replicate and extend previous work showing that the alliance did not mediate the effect of treatment expectancies on changes in anxiety in the UP (Sauer-Zavala et al., 2018). Together, these results suggest that the alliance may have a smaller direct impact on clinical outcomes than skillfulness in the UP. Given the relatively greater emphasis on skill rehearsal and practice in the UP compared to other treatments, these results suggest skillfulness is a putative mechanism of action and that when patients are more skillful than normal, they are more likely to experience symptom reductions. However, because a strong alliance can enhance patients' motivation to practice skills, future researchers should explore whether the alliance indirectly influences clinical outcomes through skillfulness and other putative mechanisms. 
Between-persons, both skillfulness and the alliance were differentially related to anxiety and depression. People with more severe anxiety tended to report a stronger alliance but less skillfulness whereas people with more severe depression tended to report a weaker alliance and greater skillfulness. Of note, when examining the skillfulness items with the largest betweenperson effect sizes, people with more severe depression tended to report using skills more frequently but less effectively than those with less severe depression. Although the only statistically significant between-person relation was between depression severity and the alliance, all between-person effects were small-to-medium sized. These results replicate previous research demonstrating mixed relations between anxiety and the alliance (Luong et al., 2020) but consistent negative relations between depression and the alliance (Flückiger et al., 2018) and extend research on skillfulness and emotional outcomes. Together, these results suggest that people with more severe anxiety view the alliance with their therapist more strongly, even if they have more difficulties implementing skills than those with less severe anxiety, whereas people with more severe depression have more difficulties establishing a strong alliance with their therapist and try skills more frequently but are less effective at using them than those with less severe depression. By recruiting a sample of people with mixed anxiety and depressive features, we were able to test these relations parsimoniously in a single model, and we encourage future researchers to replicate these findings in larger transdiagnostic samples.

When examining the effects of UP skills in Aim 2, more frequent use of UP skills than one's personal average predicted small-sized decreases in anxiety but not depression. These results build on previous findings regarding the effectiveness of mindfulness skills for anxiety (Boswell et al., 2014; Conklin et al., 2015; Sauer-Zavala et al., 2021) and extends these findings to UP skills more generally. More frequent use of UP skills may predict decreases in anxiety by 
preventing patients from engaging in avoidance prompted by this emotion and helping them learn more rapidly that they can tolerate the anxiety. Because no specific skills predicted changes in anxiety, these results suggest frequent, potentially idiographic, skill use may be most beneficial for anxiety. That is, when targeting anxiety, therapists may be encouraged to focus on how frequently patients use their skills regardless of the skill(s) being practiced and utilize patient feedback about which skills they are willing to practice more frequently.

By contrast, practicing alternative actions to avoidant urges (Countering Emotional Behaviors 2) more frequently than one's personal average predicted decreases in depression but not anxiety, conceptually replicating a single-case experimental design with this skill (SauerZavala et al., 2020). Alternative actions are similar to behavioral activation, which has repeatedly been shown to predict decreases in depression (Cuijpers et al., 2021; Webb et al., 2016), in that alternative actions allow patients to engage in activities they want to avoid but that may be important to them, promoting a sense of mastery or pleasure. These results suggest that alternative actions may demonstrate relatively unique effects on depression.

Although more frequent use of alternative actions (Countering Emotional Behaviors 2) predicted decreased depression, identifying cognitive, physical, and behavioral aspects of emotions (Understanding Emotions 1) more frequently than one's personal average predicted increases in depression but not anxiety. Given this item was written to be understood outside of the context of this particular module, it is possible patients interpreted it more as rumination, or thinking over and over about maladaptive aspects of their emotions. Given the reciprocal relation between rumination and depression (Whisman et al., 2020), it is not surprising that more frequent use of this skill would predict increases in depression. These results suggest therapists should clearly specify how to productively identify aspects of emotions without ruminating on 
them so patients can distinguish emotion identification from rumination.

Between-persons, people with more severe anxiety tended to use Confronting Physical Sensations less and both Cognitive Flexibility and Countering Emotional Behaviors 1 (identifying avoidant behaviors) more than those with less severe anxiety. These results suggest that people with more severe anxiety were less likely to practice behavioral skills that elicited physical symptoms of anxiety and more likely to practice cognitive skills to take note of and think about their experiences differently, consistent with a more avoidant approach to emotions. By contrast, people with more severe depression tended to use both Confronting Physical Sensations and Understanding Emotions 1 more and Understanding Emotions 2 less than those with less severe depression. These results suggest that people with more severe depression identify and feel their immediate emotional and physical symptoms but are less likely to compare the short- and long-term consequences of their behaviors. Although only the relation between Confronting Physical Sensations and anxiety severity was statistically significant, these effects were all small-to-medium sized, so we encourage replications with larger samples.

Of note, there was limited evidence of reverse causality. More severe experiences of anxiety predicted increases in identifying avoidant behaviors (Countering Emotional Behaviors 1), and more severe experiences of depression predicted increases in Confronting Physical Sensations, suggesting greater anxiety may promote greater attention to one's avoidant behaviors and greater depression may lead people to resist their physical sensations less forcefully. Because these were the only skills predicted by symptom change, the pattern of results suggests more evidence for a unidirectional relation between skillfulness and use predicting symptom change.

The results of this study should be considered in light of its limitations. These results apply most consistently to the early sessions of transdiagnostic CBT, which reduced our power 
to detect within-person effects and may not always generalize to full courses of care (Tables S21-S37). However, given that the majority of symptom change tends to occur relatively early in treatment, these results speak to a particularly potent treatment window. Second, although we hypothesized general patterns of effects, particularly in Aim 1, the results from Aim 2 should be considered exploratory and in need of replication. Third, although we measured outcomes relatively frequently before and after each session, participants still may have been biased on their reports of skill use, given that these were completed prior to each session. We encourage future researchers to incorporate EMA reporting of skill use into treatment designs to capture patients' behaviors as they unfold. Fourth, all measures were self-reported and therapists were able to view their patients' alliance ratings, which may have led to more socially desirable responding. Fifth, the sample was relatively small and homogeneous in terms of gender, racial background, SES, and sexual identity so these results may not generalize as well to those with different demographic characteristics, and we encourage future researchers to replicate our findings. Finally, patients received core modules of the UP rather than the full treatment package. Although delivering the UP in this way is feasible and acceptable (Sauer-Zavala et al., 2019), this design limits how well these results compare to studies of full courses of the UP.

Despite these limitations, our results highlight the importance of skill use for symptom outcomes in the UP. Within-person reductions in anxiety were predicted by greater transtheoretical skillfulness and more frequent UP skill use, and within-person reductions in depression were predicted by greater transtheoretical skillfulness and more frequent use of alternative actions. Together, these results offer specific mechanistic targets for therapists depending on their outcome of interest and characterize both transtheoretical and UP-specific mechanisms of change to develop more process-based treatments. 


\section{References}

Adler, A. D., Strunk, D. R., \& Fazio, R. H. (2015). What changes in cognitive therapy for depression? An examination of cognitive therapy skills and maladaptive beliefs. Behavior Therapy, 46(1), 96-109. https://doi.org/10.1016/j.beth.2014.09.001

Altman, A. D., Shapiro, L. A., \& Fisher, A. J. (2020). Why does therapy work? An idiographic approach to explore mechanisms of change over the course of psychotherapy using digital assessments. Frontiers in Psychology, 11, 782. https://doi.org/10.3389/fpsyg.2020.00782

American Psychiatric Association. (2013). Diagnostic and statistical manual of mental disorders (5th ed.). https://doi.org/10.1176/appi.books.9780890425596

Barlow, D. H., Sauer-Zavala, S., Farchione, T. J., Latin, H., Ellard, K. K., Bullis, J. R., Bentley, K. H., Boettcher, H. T., \& Cassiello-Robbins, C. (2018). Unified protocol for the transdiagnostic treatment of emotional disorders: Patient workbook (2nd ed.). Oxford University Press.

Bentley, K. H., Gallagher, M. W., Carl, J. R., \& Barlow, D. H. (2014). Development and validation of the Overall Depression Severity and Impairment Scale. Psychological Assessment, 26(3), 815-830. https://doi.org/10.1037/a0036216

Boswell, J. F., Anderson, L. M., \& Barlow, D. H. (2014). An idiographic analysis of change processes in the unified transdiagnostic treatment of depression. Journal of Consulting and Clinical Psychology, 82(6), 1060-1071. https://doi.org/10.1037/a0037403

Cassiello-Robbins, C., Southward, M. W., Tirpak, J. W., \& Sauer-Zavala, S. (2020). A systematic review of Unified Protocol applications with adult populations: Facilitating widespread dissemination via adaptability. Clinical Psychology Review, 78, 101852. 


\section{https://doi.org/10.1016/j.cpr.2020.101852}

Chadwick, P., Hember, M., Symes, J., Peters, E., Kuipers, E., \& Dagnan, D. (2008). Responding mindfully to unpleasant thoughts and images: Reliability and validity of the Southampton Mindfulness Questionnaire (SMQ). British Journal of Clinical Psychology, 47(Pt 4), 451-455. https://doi.org/10.1348/014466508X314891

Cuijpers, P., Quero, S., Noma, H., Ciharova, M., Miguel, C., Karyotaki, E., Ciprani, A., Cristea, I. A., \& Furukawa, T. A. (2021). Psychotherapies for depression: A network metaanalysis covering efficacy, acceptability and long-term outcomes of all main treatment types. World Psychiatry, 20(2), 283-293. https://doi.org/10.1002/wps.20860

Curran, P. J., \& Bauer, D. J. (2011). The disaggregation of within-person and between-person effects in longitudinal models of change. Annual Review of Psychology, 62, 583-619. https://doi.org/10.1146/annurev.psych.093008.100356

Eustis, E. H., Cardona, N., Nauphal, M., Sauer-Zavala, S., Rosellini, A. J., Farchione, T. J., \& Barlow, D. H. (2020). Experiential avoidance as a mechanism of change across cognitive-behavioral therapy in a sample of participants with heterogeneous anxiety disorders. Cognitive Therapy and Research, 44(2), 275-286. https://doi.org/10.1007/s10608-019-10063-6

Faul, F., Erdfelder, E., Buchner, A., \& Lang, A. G. (2009). Statistical power analyses using G*Power 3.1: Tests for correlation and regression analyses. Behavior Research Methods, 41(4), 1149-1160. https://doi.org/10.3758/BRM.41.4.1149

Flückiger, C., Del Re, A. C., Wampold, B. E., \& Horvath, A. O. (2018). The alliance in adult psychotherapy: A meta-analytic synthesis. Psychotherapy, 55(4), 316-340. http://dx.doi.org/10.1037/pst0000172 
Forand, N. R., Barnett, J. G., Strunk, D. R., Hindiyeh, M. U., Feinberg, J. E., \& Keefe, J. R. (2018). Efficacy of guided iCBT for depression and mediation of change by cognitive skill acquisition. Behavior Therapy, 49(2), 295-307. https://doi.org/10.1016/j.beth.2017.04.004

Gallagher, M. W., Long, L. J., Richardson, A., D'Souza, J., Boswell, J. F., Farchione, T. J., \& Barlow, D. H. (2020). Examining hope as a transdiagnostic mechanism of change across anxiety disorders and CBT treatment protocols. Behavior Therapy, 51(1), 190-202. https://doi.org/10.1016/j.beth.2019.06.001

Hatcher, R. L., \& Gillaspy, J. A. (2006). Development and validation of a revised short version of the Working Alliance Inventory. Psychotherapy Research, 16(1), 12-25. https://doi.org/10.1080/10503300500352500

Hu, L.-t., \& Bentler, P. M. (1999). Cutoff criteria for fit indexes in covariance structure analysis: Conventional criteria versus new alternatives. Structural Equation Modeling, 6(1), 1-55. https://doi.org/10.1080/10705519909540118

Jaeger, B. C., Edwards, L. J., Das, K., \& Sen, P. K. (2017). An $R^{2}$ statistic for fixed effects in the generalized linear mixed model. Journal of Applied Statistics, 44(6), 1086-1105. https://doi.org/10.1080/02664763.2016.1193725

Lafit, G., Adolf, J. K., Dejonckheere, E., Myin-Germeys, I., Viechtbauer, W., \& Ceulemans, E. (2021). Selection of the number of participants in intensive longitudinal studies: A userfriendly Shiny app and tutorial for performing power analysis in multilevel regression models that account for temporal dependencies. Advances in Methods and Practices in Psychological Science, 4(1), 1-24. https://doi.org/10.1177/2515245920978738

Luong, H. K., Drummond, S. P. A., \& Norton, P. J. (2020). Elements of the therapeutic 
relationship in CBT for anxiety disorders: A systematic review. Journal of Anxiety Disorders, 76, Article 102322. https://doi.org/10.1016/j.janxdis.2020.102322

McNeish, D., \& Stapleton, L. M. (2016). Modeling clustered data with very few clusters. Multivariate Behavioral Research, 51(4), 495-518. https://doi.org/10.1080/00273171.2016.1167008

Muthén, L. K., \& Muthén, B. O. (1998-2012). Mplus user’s guide (7th ed.). Muthén \& Muthén.

Niileksela, C. R., Ghosh, A., \& Janis, R. A. (2021). The dose-effect and good enough level models of change for specific psychological concerns. Journal of Consulting and Clinical Psychology, 89(3), 200-213. https://doi.org/10.1037/ccp0000635

Norman, S. B., Cissell, S. H., Means-Christensen, A. J., \& Stein, M. B. (2006). Development and validation of an Overall Anxiety Severity and Impairment Scale (OASIS). Depression and Anxiety, 23(4), 245-249. https://doi.org/10.1002/da.20182

Sakiris, N., \& Berle, D. (2019). A systematic review and meta-analysis of the Unified Protocol as a transdiagnostic emotion regulation based intervention. Clinical Psychology Review, 72, 101751. https://doi.org/10.1016/j.cpr.2019.101751

Sauer-Zavala, S., Boswell, J. F., Bentley, K. H., Thompson-Hollands, J., Farchione, T. J., \& Barlow, D. H. (2018). Expectancies, working alliance, and outcome in transdiagnostic and single diagnosis treatment for anxiety disorders: An investigation of mediation. Cognitive Therapy and Research, 42(2), 135-145. https://doi.org/10.1007/s10608-017$\underline{9855-8}$

Sauer-Zavala, S., Cassiello-Robbins, C., Ametaj, A. A., Wilner, J. G., \& Pagan, D. (2019). Transdiagnostic treatment personalization: The feasibility of ordering Unified Protocol modules according to patient strengths and weaknesses. Behavior Modification, 43(4), 


\section{8-543. https://doi.org/10.1177/0145445518774914}

Sauer-Zavala, S., Cassiello-Robbins, C., Woods, B. K., Curreri, A., Wilner Tirpak, J., \&

Rassaby, M. (2020). Countering emotional behaviors in the treatment of borderline personality disorder. Personality Disorders: Theory, Research, \& Treatment, 11(5), 328338. https://doi.org/10.1037/per0000379

Sauer-Zavala, S., Rosellini, A. J., Bentley, K. H., Ametaj, A. A., Boswell, J. F., CassielloRobbins, C., Wilner Tirpak, J., Farchione, T. J., \& Barlow, D. H. (2021). Skill acquisition during transdiagnostic treatment with the Unified Protocol. Behavior Therapy. Advance online publication. https://doi.org/10.1016/j.beth.2021.03.001

Sauer-Zavala, S., Southward, M. W., Stumpp, N. E., Semcho, S. A., Hood, C. O., Garlock, A., \& Urs, A. (2021). A SMART approach to personalized care: How to select and sequence skills in transdiagnostic CBT. Manuscript submitted for publication.

Sauer-Zavala, S., Wilner, J. G., Cassiello-Robbins, C., Saraff, P., \& Pagan, D. (2019). Isolating the effect of opposite action in borderline personality disorder: A laboratory-based alternating treatment design. Behaviour Research and Therapy, 117, 79-86.

\section{https://doi.org/10.1016/j.brat.2018.10.006}

Southward, M. W., Eberle, J. W., \& Neacsiu, A. D. (2021a). Multilevel associations of daily skill use and effectiveness with anxiety, depression, and stress in a transdiagnostic sample undergoing Dialectical Behavior Therapy skills training. Cognitive Behaviour Therapy. Advance online publication. https://doi.org/10.1080/16506073.2021.1907614

Southward, M. W., \& Sauer-Zavala, S. (2020). Experimental manipulations to test theory-driven mechanisms of cognitive behavior therapy. Frontiers in Psychiatry, 11, Article 603009. https://doi.org/10.3389/fpsyt.2020.603009 
Southward, M. W., Sauer-Zavala, S., \& Cheavens, J. S. (2021b). Specifying the mechanisms and targets of emotion regulation: A translational framework from affective science to psychological treatment. Clinical Psychology: Science and Practice, 28(2), 168-182. https://doi.org/10.1037/cps0000003

Steiger, J. H. (1980). Tests for comparing elements of a correlation matrix. Psychological Bulletin, 87(2), 245-251. https://doi.org/10.1037/0033-2909.87.2.245

Wang, L. P., \& Maxwell, S. E. (2015). On disaggregating between-person and within-person effects with longitudinal data using multilevel models. Psychological Methods, 20(1), 63-83. https://doi.org/10.1037/met0000030

Webb, C. A., Beard, C., Kertz, S. J., Hsu, K. J., \& Björgvinsson, T. (2016). Differential role of CBT skills, DBT skills and psychological flexibility in predicting depressive versus anxiety symptom improvement. Behaviour Research and Therapy, 81, 12-20. https://doi.org/10.1016/j.brat.2016.03.006

Whisman, M. A., du Pont, A., \& Butterworth, P. (2020). Longitudinal associations between rumination and depressive symptoms in a probability sample of adults. Journal of Affective Disorders, 260, 680-686. https://doi.org/10.1016/j.jad.2019.09.035

Wilamowska, Z. A., Thompson-Hollands, J., Fairholme, C. P., Ellard, K. K., Farchione, T. J., \& Barlow, D. H. (2010). Conceptual background, development, and preliminary data from the unified protocol for transdiagnostic treatment of emotional disorders. Depression and Anxiety, 27(10), 882-890. https://doi.org/10.1002/da.20735

Woods, B. K., Sauer-Zavala, S., Farchione, T. J., \& Barlow, D. H. (2020). Isolating the effects of mindfulness training across anxiety disorder diagnoses in the Unified Protocol. Behavior Therapy, 51(6), 972-983. https://doi.org/10.1016/j.beth.2020.01.001 


\section{Table 1a}

Factor Loadings of Dimensions of Skillfulness and Use Scale Items

\section{Factor}

\begin{tabular}{lcc} 
Item & $\begin{array}{c}\text { Within-Person } \\
\lambda(S E)\end{array}$ & $\begin{array}{c}\text { Between-Person } \\
\lambda(S E)\end{array}$ \\
\hline 1. Knowledge & $.51(.07)$ & $.67(.13)$ \\
2. Frequency & $.63(.06)$ & $.75(.07)$ \\
3. Quality & $.76(.06)$ & $1.00(.02)$ \\
4. Effectiveness & $.74(.04)$ & $.99(.02)$ \\
\hline
\end{tabular}

Note. $\chi^{2}(4)=8.17, p=.09 ; \mathrm{RMSEA}=.054 ; \mathrm{CFI}=$ $.989 ;$ TLI $=.967 ;$ SRMR between $=.020$, within $=$

.028. Loadings represent fully standardized solution.

All factor loadings are significant, $p$ s $<.01$.

\section{Table 1b}

Factor Loadings of Unified Protocol Skill Use Scale Items

\begin{tabular}{lcc}
\multirow{2}{*}{ Item } & Within-Person & \multicolumn{2}{c}{ Factor } \\
$\lambda(S E)$ & $\begin{array}{c}\text { Between-Person } \\
\lambda(S E)\end{array}$ \\
\hline Understanding Emotions 1 & $.68(.05)$ & $.94(.03)$ \\
Understanding Emotions 2 & $.66(.05)$ & $.94(.03)$ \\
Mindful Emotion Awareness & $.63(.05)$ & $.88(.03)$ \\
Cognitive Flexibility & $.57(.07)$ & $.91(.07)$ \\
Countering Emotional Behaviors 1 & $.54(.06)$ & $.95(.03)$ \\
Countering Emotional Behaviors 2 & $.70(.05)$ & $.94(.03)$ \\
Confronting Physical Sensations & $.51(.07)$ & $.95(.04)$ \\
\hline
\end{tabular}

Note. $\chi^{2}(28)=57.00, p<.01 ; \mathrm{RMSEA}=.055 ; \mathrm{CFI}=.950 ; \mathrm{TLI}=.926 ;$ SRMR between $=.026$, within $=.055$. Loadings represent fully standardized solution. Understanding Emotions 1 = identifying cognitive, physical, and behavioral features of emotions. Understanding Emotions 2 = identifying short- and longterm consequences of behaviors. Mindful Emotion Awareness = nonjudgmental present-moment awareness. Cognitive Flexibility $=$ considering alternative interpretations for one's thoughts. Countering Emotional Behaviors $1=$ identifying avoidant behaviors. Countering Emotional Behaviors $2=$ adaptive alternative actions to avoidant urges. Confronting Physical Sensations $=$ feeling uncomfortable physical sensations without avoiding or escaping. All factor loadings are significant, $p s<.01$. 
Table 2a

Correlations Among Variables of Interest - Aim 1

\begin{tabular}{lrrrrrr} 
Variable & \multicolumn{1}{c}{1} & \multicolumn{1}{c}{2} & \multicolumn{1}{c}{3} & \multicolumn{1}{c}{4} & \multicolumn{1}{c}{5} & \multicolumn{1}{c}{6} \\
\hline 1. Skillfulness & & $.40 * *$ & $.31 * *$ & $-.22 * *$ & $-.29 * *$ & $.44 * *$ \\
2. Cognitive Skills & $.57 * *$ & & $.45^{* *}$ & $-.31 * *$ & $-.28 * *$ & $.27 * *$ \\
3. Mindfulness & .09 & $.27 *$ & & $-.30 * *$ & $-.36 * *$ & $.20 * *$ \\
4. Anxiety & -.05 & -.20 & $-.38 * *$ & & $.42 * *$ & $-.14 *$ \\
5. Depression & .04 & -.11 & -.19 & $.64 * *$ & & $-.23 * *$ \\
6. Alliance & $.54 * *$ & $.26 *$ & .01 & -.09 & -.18 &
\end{tabular}

Note. Between-person correlations are below the diagonal, and within-person correlations are above the diagonal. Skillfulness $=$ Dimensional Skillfulness and Use Scale. Cognitive Skills = Unified Protocol - Cognitive Skills Questionnaire. Mindfulness = Southampton Mindfulness Questionnaire. Anxiety = Overall Anxiety Severity and Impairment Scale. Depression = Overall Depression Severity and Impairment Scale. Alliance = Working Alliance Inventory - Short Revised.

$* p<.05, * * p<.01$. 


\section{Table 2b}

Correlations Among Variables of Interest - Aim 2

\begin{tabular}{|c|c|c|c|c|c|c|c|c|c|c|c|c|c|c|}
\hline Variable & 1 & 2 & 3 & 4 & 5 & 6 & 7 & 8 & 9 & 10 & 11 & 12 & 13 & 14 \\
\hline 1. UP Skill Frequency & & $.31 * *$ & $.69 * *$ & $.71^{* *}$ & $.72 * *$ & $.68^{* *}$ & $.57 * *$ & $.75 * *$ & $.59 * *$ & $.44 * *$ & $.27 * *$ & $-.30 * *$ & $-.28 * *$ & $.28 * *$ \\
\hline 2. UP Skill Quantity & .07 & & $.22 * *$ & $.28^{* *}$ & $.27 * *$ & $.27 * *$ & $.33^{* *}$ & $.36^{* *}$ & $.21 *$ & $.30^{* *}$ & $.25^{* *}$ & $-.23 * *$ & $-.21 * *$ & $.36 * *$ \\
\hline 3. Understanding Emotions 1 & $.89 * *$ & .12 & & $.52 * *$ & $.45^{* *}$ & $.42 * *$ & $.24 * *$ & $.38^{* *}$ & $.27 * *$ & $.33 * *$ & $.18^{* * *}$ & $-.21 * *$ & -.10 & $.22 * *$ \\
\hline 4. Understanding Emotions 2 & $.88 * *$ & .29 & $.77 * *$ & & $.49 * *$ & $.42 * *$ & $.21 * *$ & $.42 * *$ & $.25 * *$ & $.26^{* *}$ & $.21 * *$ & $-.28 * *$ & $-.27 * *$ & $.35 * *$ \\
\hline 5. Mindful Emotion Awareness & $.76^{* * *}$ & -.10 & $.67^{* *}$ & $.59^{* *}$ & & $.37^{* *}$ & $.23^{* *}$ & $.35^{* *}$ & $.42 * *$ & $.24 * *$ & $.21^{* *}$ & $-.31 * *$ & $-.25 * *$ & $.29 * *$ \\
\hline 6. Cognitive Flexibility & $.77^{* *}$ & .06 & $.58^{* *}$ & $.71^{* *}$ & $.51^{* *}$ & & $.26^{* *}$ & $.43^{* *}$ & $.27 * *$ & $.28 * *$ & $.21^{* *}$ & $-.18 * *$ & $-.21 * *$ & $.21 * *$ \\
\hline 7. Countering Emotional Behaviors 1 & $.79^{* *}$ & $.41^{* *}$ & $.69^{* *}$ & $.68^{* *}$ & $.58^{* *}$ & $.54^{* *}$ & & $.46^{* *}$ & .15 & $.16^{*}$ & $.25^{* *}$ & -.08 & .02 & $.17 *$ \\
\hline 8. Countering Emotional Behaviors 2 & $.76^{* *}$ & .04 & $.62 * *$ & $.60 * *$ & $.46^{* *}$ & $.57 * *$ & $.67 * *$ & & $.34 * *$ & $.22 * *$ & $.27 * *$ & $-.34 * *$ & $-.39 * *$ & .07 \\
\hline 9. Confronting Physical Sensations & $.84^{* *}$ & .05 & $.80^{* *}$ & $.72 * *$ & $.62^{* *}$ & $.50^{* *}$ & $.62 * *$ & $.53^{* *}$ & & $.21^{* *}$ & $.14^{*}$ & $-.19^{*}$ & -.15 & $.28 * *$ \\
\hline 10. Cognitive Skills & $.64 * *$ & .09 & $.57^{* *}$ & $.53^{* *}$ & $.34 *$ & $.60^{* *}$ & $.54 * *$ & $.52^{* *}$ & $.46^{* *}$ & & $.45^{* *}$ & $-.31 * *$ & $-.28 * *$ & $.23 * *$ \\
\hline 11. Mindfulness & .12 & .01 & .05 & .10 & .11 & .21 & .01 & .08 & .12 & $.27^{*}$ & & $-.30 * *$ & $-.36 * *$ & $.25 * *$ \\
\hline 12. Anxiety & $<-.01$ & .14 & -.02 & $<.01$ & .12 & .12 & .27 & -.11 & -.10 & -.20 & $-.38 * *$ & & $.42 * *$ & -.11 \\
\hline 13. Depression & .14 & $.31 *$ & .18 & .06 & .11 & .16 & $.32 *$ & .01 & .23 & -.11 & -.19 & $.64 * *$ & & $-.12 *$ \\
\hline 14. Alliance & $.45^{* *}$ & $.31 *$ & $.43 *$ & $.54 * *$ & $.41 * *$ & .29 & $.48^{* *}$ & $.40^{* *}$ & .30 & $.26^{*}$ & .01 & -.09 & -.18 & \\
\hline
\end{tabular}

Note. Between-person correlations are below the diagonal, and within-person correlations are above the diagonal. UP $=$ Unified Protocol. UP Skill Frequency $=$ Average

frequency with which all skills were used. UP Skill Quantity $=$ Total number of skills used. Understanding Emotions $1=$ identifying cognitive, physical, and behavioral features of emotions. Understanding Emotions 2 = identifying short- and long-term consequences of behaviors. Mindful Emotion Awareness = nonjudgmental present-moment awareness. Cognitive Flexibility = considering alternative interpretations for one's thoughts. Countering Emotional Behaviors $1=$ identifying avoidant behaviors. Countering Emotional Behaviors 2 = adaptive alternative actions to avoidant urges. Confronting Physical Sensations $=$ feeling uncomfortable physical sensations without avoiding or escaping. Cognitive Skills $=$ Unified Protocol - Cognitive Skills Questionnaire. Mindfulness $=$ Southampton Mindfulness Questionnaire. Anxiety $=$ Overall Anxiety Severity and Impairment Scale. Depression = Overall Depression Severity and Impairment Scale. Alliance $=$ Working Alliance Inventory-Short Revised.

$* p<.05, * * p<.01$. 


\section{Table 3a}

Session-by-Session Means and Standard Deviations of Primary Variables of Interest

\begin{tabular}{|c|c|c|c|c|c|c|c|}
\hline Variable & $\begin{array}{l}\text { Session } 1 \\
(n=68) \\
M(S D) \\
\end{array}$ & $\begin{array}{l}\text { Session } 2 \\
(n=63) \\
M(S D) \\
\end{array}$ & $\begin{array}{l}\text { Session } 3 \\
(n=61) \\
M(S D) \\
\end{array}$ & $\begin{array}{l}\text { Session } 4 \\
(n=60) \\
M(S D) \\
\end{array}$ & $\begin{array}{l}\text { Session } 5 \\
(n=59) \\
M(S D) \\
\end{array}$ & $\begin{array}{c}\text { Session } 6 \\
(n=59) \\
M(S D) \\
\end{array}$ & $\begin{array}{c}\text { Session/Week } 7 \\
(n=55) \\
M(S D) \\
\end{array}$ \\
\hline Skillfulness & - & $7.41(2.61)$ & $7.20(2.73)$ & $7.30(2.85)$ & $7.41(2.86)$ & $8.03(2.81)$ & $7.53(3.04)$ \\
\hline Frequency & - & $2.26(1.16)$ & $2.11(1.13)$ & $2.07(1.02)$ & $2.27(1.08)$ & $2.24(1.06)$ & $2.31(1.10)$ \\
\hline Quality & - & $1.56(.80)$ & $1.52(.77)$ & $1.62(.78)$ & $1.59(.79)$ & $1.83(.73)$ & $1.60(.81)$ \\
\hline Anxiety & $8.53(3.53)$ & $8.03(3.35)$ & $7.93(3.65)$ & $7.75(3.40)$ & $7.03(3.07)$ & $7.08(3.57)$ & $6.55(3.54)$ \\
\hline Depression & $7.49(4.78)$ & $6.70(4.57)$ & $6.41(4.66)$ & $5.93(4.01)$ & $5.85(4.59)$ & $5.03(4.51)$ & $4.91(4.09)$ \\
\hline Alliance & $47.62(8.43)$ & $51.17(7.04)$ & $51.48(7.71)$ & $52.45(6.53)$ & $54.52(5.06)$ & $53.67(7.26)$ & - \\
\hline
\end{tabular}

Note. Skillfulness = Dimensions of Skillfulness and Use Scale. Anxiety = Overall Anxiety Severity and Impairment Scale. Depression $=$ Overall Depression Severity and Impairment Scale. Alliance $=$ Working Alliance Inventory - Short Revised.

\section{Table 3b}

Session-by-Session Means and Standard Deviations of UP Skill Use Scale

\begin{tabular}{lcccccc} 
& $\begin{array}{c}\text { Session 2 } \\
(n=34)\end{array}$ & $\begin{array}{c}\text { Session 3 } \\
(n=36)\end{array}$ & $\begin{array}{c}\text { Session 4 } \\
(n=38)\end{array}$ & $\begin{array}{c}\text { Session 5 } \\
(n=37)\end{array}$ & $\begin{array}{c}\text { Session 6 } \\
(n=39)\end{array}$ & $\begin{array}{c}\text { Session/Week 7 } \\
(n=37)\end{array}$ \\
Variable & $M(S D)$ & $M(S D)$ & $M(S D)$ & $M(S D)$ & $M(S D)$ & $M(S D)$ \\
\hline Frequency & $2.35(1.06)$ & $2.47(.99)$ & $2.72(.81)$ & $2.74(.94)$ & $2.84(1.05)$ & $2.79(.97)$ \\
Understanding Emotions 1 & $2.75(1.23)$ & $2.82(1.13)$ & $3.11(1.05)$ & $3.17(.97)$ & $3.08(1.10)$ & $3.11(1.15)$ \\
Understanding Emotions 2 & $2.11(1.22)$ & $2.27(1.21)$ & $2.60(1.22)$ & $2.69(1.13)$ & $2.68(1.28)$ & $2.84(1.28)$ \\
Mindful Emotion Awareness & $2.40(1.26)$ & $2.28(1.31)$ & $2.51(1.07)$ & $2.47(1.14)$ & $3.03(1.24)$ & $2.80(1.16)$ \\
Cognitive Flexibility & $2.48(1.19)$ & $2.41(1.24)$ & $2.64(1.25)$ & $2.56(1.21)$ & $2.95(1.29)$ & $2.89(1.28)$ \\
Countering Emotional Behaviors 1 & $2.38(1.56)$ & $2.61(1.42)$ & $2.90(1.34)$ & $2.83(1.21)$ & $2.85(1.40)$ & $2.86(1.31)$ \\
Countering Emotional Behaviors 2 & $1.68(1.28)$ & $2.19(1.33)$ & $2.48(1.33)$ & $2.63(1.43)$ & $2.52(1.48)$ & $2.39(1.32)$ \\
Confronting Physical Sensations & $2.29(1.55)$ & $2.92(1.44)$ & $2.74(1.14)$ & $2.79(1.23)$ & $2.85(1.37)$ & $2.53(1.21)$
\end{tabular}

Note. Frequency $=$ Average frequency with which all skills were used. Understanding Emotions $1=$ identifying cognitive, physical, and behavioral features of emotions. Understanding Emotions 2 = identifying short- and long-term consequences of behaviors. Mindful Emotion Awareness = nonjudgmental present-moment awareness. Cognitive Flexibility = considering alternative interpretations for one's thoughts. Countering Emotional Behaviors $1=$ identifying avoidant behaviors. Countering Emotional Behaviors 2 = adaptive alternative actions to avoidant urges. Confronting Physical Sensations $=$ feeling uncomfortable physical sensations without avoiding or escaping. 


\section{Appendix}

The data reported in this manuscript have been previously published and were collected as part of a larger data collection effort in a randomized clinical trial. Findings from the data collection have been reported in two separate manuscripts. MS1 (Southward \& Sauer-Zavala, 2020) includes measures of anxiety symptoms (OASIS) from three participants at nonoverlapping time points with the current manuscript. MS2 (Sauer-Zavala et al., 2021) reports on changes in anxiety (OASIS) and depression (ODSIS) between the three arms of the randomized clinical trial but does not include an overall test of changes in these measures collapsing across arms. 